\title{
Antireflection coatings on starshade optical edges for solar glint suppression
}

\author{
Dylan McKeithen $\odot$, ${ }^{\text {a,* }}$ Stuart Shaklan $\odot,{ }^{\text {a }}$ David Sheikh $\odot,{ }^{\mathrm{b}}$ \\ Kunjithapatham Balasubramanian, ${ }^{a}$ and Eric Lowe ${ }^{\text {a,c }}$ \\ a Jet Propulsion Laboratory, California Institute of Technology, Pasadena, California, \\ United States \\ ${ }^{\mathrm{b}} \mathrm{ZeCoat}$ Corporation, Granite City, Illinois, United States \\ ${ }^{c}$ Morrissey College of Arts and Sciences, Boston College, Chestnut Hill, Massachusetts, \\ United States
}

\begin{abstract}
Starshades are designed to enable the direct observation of an exoplanet by blocking the light of the planet's star from reaching the telescope. As discussed in our companion paper [S. Shaklan et al., "Solar glint from uncoated starshade optical edges," J. Astron. Telesc. Instrum. Syst. 7(2), 021204 (2021)], diffraction and reflection of sunlight incident on the starshade's razor-sharp uncoated edges will appear as glint that may be brighter than the feeble light of the exoplanet. We report on the measurement and modeling of thin, conformal, multilayer antireflection coatings that reduce solar glint by more than an order of magnitude when applied to uncoated edges. We used the Lumerical finite-difference time-domain simulation software suite to determine the performance of coatings designed to work on a flat surface when applied to a sharp, curved edge. Laboratory measurements of coated edges, including a 50-cm long segment, confirm the glint reduction predicted by these models. We consider two coating approaches and compare their performance: a line-of-sight coating and a coating that uniformly covers the entire terminal edge. Starting with a wide range of coating designs emphasizing different angles of incidence and bandpass characteristics, we use Lumerical to account for edge diffraction and reflection, and we optimize the designs for the Starshade Rendezvous Mission and the HabEx mission concept.

(C) The Authors. Published by SPIE under a Creative Commons Attribution 4.0 Unported License. Distribution or reproduction of this work in whole or in part requires full attribution of the original publication, including its DOI. [DOI: 10.1117/1.JATIS.7.2.021208]
\end{abstract}

Keywords: starshade; AR coatings; glint; exoplanet; finite-difference time-domain.

Paper 20115SS received Aug. 1, 2020; accepted for publication Dec. 1, 2020; published online Jan. 8, 2021.

\section{Introduction}

A starshade mission is a large, deployed, flower-shaped opaque screen flown in tandem with a space telescope to enable direct imaging of nearby exoplanets. ${ }^{1}$ The starshade's petals are designed to suppress the diffraction of starlight at the $10^{-10}$ level, enabling the telescope to image exoplanets as close as tens of milliarcseconds from their parent stars. Several mission design concepts have been proposed, including the 26-m diameter Roman Space Telescope Starshade Rendezvous Mission (SRM) ${ }^{2-4}$ and the 52-m diameter Habitable Exoplanet (HabEx) Observatory Starshade. ${ }^{5,6}$

During observations, glint from the Sun appears along the starshade's specularly reflecting edges and is mainly concentrated in two bright lobes interior to the petal tips (Fig. 1). The glint is a combination of reflection and diffraction along the petal edges. ${ }^{7}$ The Exoplanet Exploration Program's starshade technology development effort has the goal of demonstrating that the glint is fainter than a visual magnitude of $V=25,{ }^{8}$ so it does not overwhelm the exoplanet light. It is highly desirable to further reduce the brightness so that the glint becomes a minor contributor of noise when observing the faintest planets.

*Address all correspondence to Dylan McKeithen, dylan.m.mckeithen@jpl.nasa.gov 


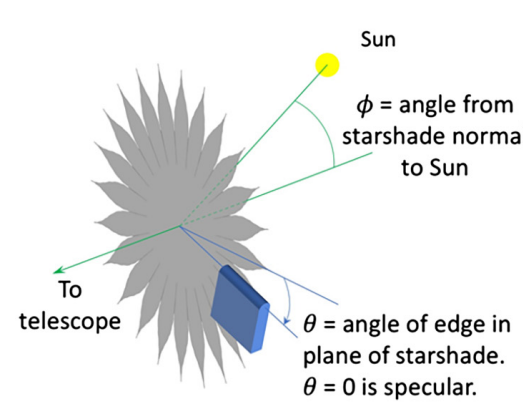

(a)

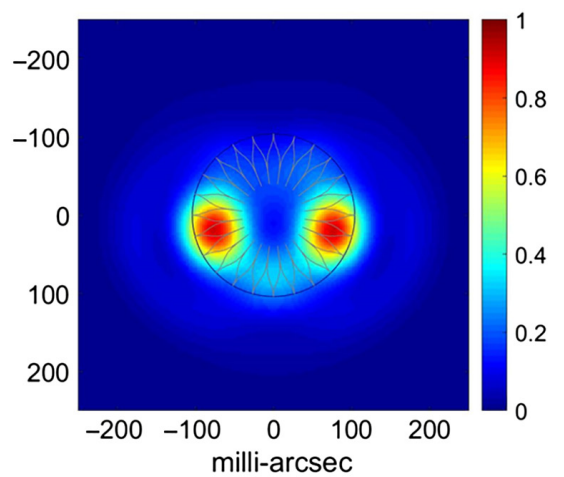

(b)

Fig. 1 (a) The Sun appears to the side and behind the starshade, which is directly between the star and telescope. Glint is brightest along edges oriented for specular reflection of the sunlight. (b) Glint appears in the form of two lobes, shown here for a bandpass of 615 to $800 \mathrm{~nm}$ with the SRM starshade positioned $26 \mathrm{Mm}$ from the telescope and the Sun behind and below the page. The starshade outline is shown for scale.

The brightness of the solar glint lobes is heavily dependent on the scattering properties of the optical edge, which comprises the perimeter of the starshade. It has been shown that an amorphous metal (AM) iron-nickel alloy foil with a sharp, etched edge (radius $\sim 150 \mathrm{~nm}$ ) has the ability to control the glint to a visual magnitude of $V \sim 25$ for the SRM and $V \sim 27$ for the HabEx mission concept. ${ }^{9}$ The application of an antireflective (AR) coating to the optical edge can reduce the scatter by minimizing the reflection component and destructively interfering or apodizing the diffraction component. The design is challenging, however, because both geometrical reflection at highly curved surfaces and diffraction from subwavelength diameter structures are in play.

A candidate AR coating needs to satisfy several criteria to be considered. The coating process must be scalable and allow for the coating to be applied to optical edge segments roughly $1 \mathrm{~m}$ in length. The coating itself must also be thin to limit the scattering surface area of the optical edge. In addition, the coating must be robust and capable of surviving the temperature extremes and radiation environments in space. Finally, the coating should be cleanable without causing damage to the coating or optical edge. The thickness of the coating, even if several microns, is negligible compared with the overall size of the starshade and has no measurable effect on the formation of the stellar shadow; the coating only affects solar glint.

In this study, the nominal radius of the terminal edge is set to $150 \mathrm{~nm}$ to approximate the measured terminal radius of the etched coupons. ${ }^{10}$ A typical scanning electron microscope image is reproduced here in Fig. 2, revealing a terminal edge with structural variability on a

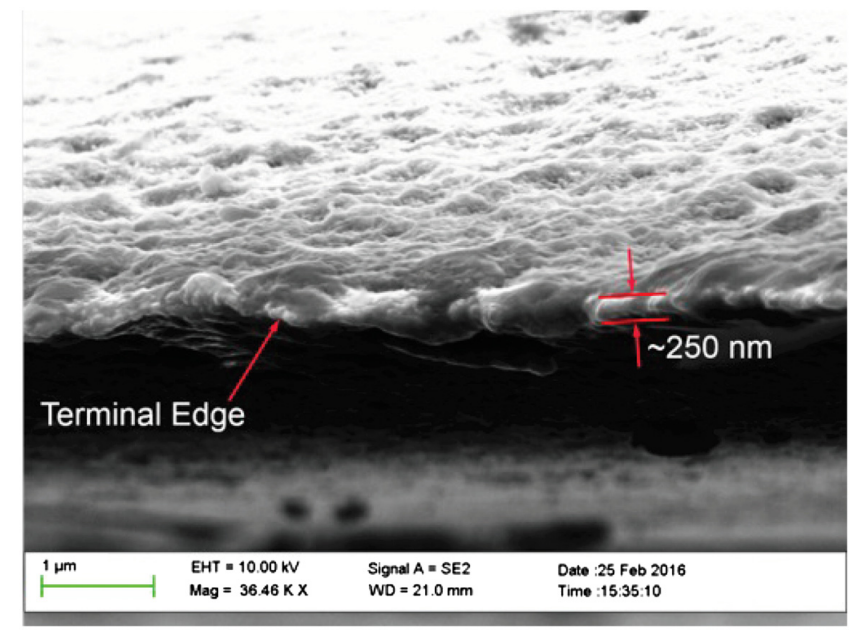

Fig. 2 SEM image of AM terminal edge. Reproduced with permission from Ref. 10. 
Table 1 Mission bands and Sun angles

\begin{tabular}{lccc}
\hline \hline & \multicolumn{2}{c}{ SRM } & \\
\cline { 2 - 3 } & Blue band & Green band & HabEx \\
\hline Wavelengths $(\mathrm{nm})$ & $425-552$ & $615-800$ & $300-1000$ \\
Sun angles $^{\mathrm{a}}$ & $54-83 \mathrm{deg}$ & $54-83 \mathrm{deg}$ & $40-85 \mathrm{deg}$ \\
Distance $^{\mathrm{b}}(\mathrm{Mm})$ & 37.24 & 25.7 & 76.6 \\
\hline \hline
\end{tabular}

${ }^{a}$ Angle subtended between the starshade-Sun direction and the starshade-target star direction.

${ }^{b}$ Nominal separation between the telescope and the starshade.

subwavelength scale. Our scatter results, based on a simple uniform-radius semicircular edge, though a crude approximation to the terminal edge, have been successful in matching experimental data. $^{7}$

Past work has studied the use of carbon nanotube-based AR coatings applied to optical edge test coupons. ${ }^{10,11}$ While these coatings are extremely dark, with total integrated reflectance of $\sim 1 \%$, the coatings are typically tens of microns thick. They greatly increase the surface area of the terminal edge, resulting in performance comparable to bare metal. Such coatings would be worthy of further consideration if they could be made thin enough, $\sim 1 \mu \mathrm{m}$, to take advantage of their intrinsic low reflectivity. Here, we consider an alternative approach using hybrid absorptive-interferometric coatings to achieve low reflectivity on the order of a few percent, using robust, proven, flight-qualified materials. The coatings are less than 500-nm thick and are conformal to the terminal edge geometry. They are designed to reduce reflectance in the visible and near-infrared bands.

The performance of the designs has been analyzed for the SRM and the HabEx mission concepts. The parameters used for these two missions are given in Table 1.

There is a large body of analytical work describing scatter from half-planes, beginning with Sommerfeld's original work on perfectly conducting, infinitesimally thin planes. ${ }^{12}$ Under these conditions, the scatter consists entirely of diffraction, with no reflection component. Thick, nonperfectly conducting planes have also been analyzed. Umul ${ }^{13}$ provided a concise list of the key papers in the field. In practice, the analyses are not suitable for the purpose of optimizing a multilayer, broadband coating applied on the curved terminal edge of a metallic substrate. Thus, we adopted the approach of commissioning a wide range of AR coatings using suitable materials as starting points for our simulation-based performance study.

In Sec. 2, we introduce the coating design and key characteristics, but we do not discuss proprietary properties, including the materials and layer thicknesses. We consider both lineof-sight (LoS) coatings that preserve the edge geometry as well as coatings that grow uniformly around the sides and tip of the edge. The coatings are designed for low reflectivity across a broad spectral band and a wide range of incident angles. The designs, however, do not consider the diffraction component of the scattered light. Section 3 describes how we model the total scatter, including diffraction combined with propagation through the curved coating, using commercial finite-difference time-domain (FDTD) simulation software. We measure the performance of four of the coatings applied at multiple angles and compared the results with FDTD predictions in Sec. 4.1. Here, we also compare the expected performance of LoS and uniform-coated edges. Next, in Sec. 5, we adjust layer thicknesses using the FDTD model to improve the performance of the best LoS and uniform-coated designs, and we report on their predicted performance and robustness to the expected manufacturing tolerances. Finally, in Sec. 6, we report on the expected solar glint lobe brightness for the two missions, and we conclude in Sec. 7.

\section{Coatings}

The goal of coating the starshade's metallic edges is to minimize the solar glint over a broadband and a large range of angles. As noted above, the coating must be robust to the space environment and suitable for cleaning without damaging its antiscatter properties. 


\subsection{Design Approach}

The designs consist of a set of absorptive-interferometric AR coatings that have low reflectivity over SRM or HabEx bands. Each design emphasizes a different combination of optimal wavelength, bandwidth, angle of incidence, and polarization properties. Several of the designs were not intended for a particular mission band, but rather were to study the effects of different reflectances and coating thicknesses on the resulting edge scatter. In a space mission, out-of-band reflectivity is limited by dichroic filters in the cameras; we are only concerned about in-band performance. ${ }^{4}$ The goal of this shotgun approach was to find a coating that best compensated for the combined diffraction and reflection from the terminal edge.

We arrived at these designs using standard thin-film design software, assuming a flat surface, a given angle of incidence, and unpolarized light. This software is blind to diffraction and curvededge reflection, yet it still resulted in coatings that greatly reduced the overall glint. Improvements to the designs accounting for edge physics with the FDTD analysis are presented in Sec. 5.

Table 2 lists the 21 coatings that we designed and analyzed. The naming scheme X:Y-Z represents the low:high ends of the targeted waveband and indexing for variations in bandpass weighting and polarization uniformity. Additionally, where it was a design parameter, a specific angle of incidence is listed. Coatings which were manufactured and tested are indicated, with results presented in Sec. 4.1.

\subsection{Reflectance and Scatter Calculation}

The reflectances listed in columns 2 and 6 of Table 2 are the average values for a uniform coating on an infinite flat substrate integrated over a range of angles and wavelengths as follows: the reflectance of each coating is averaged over the mission's waveband and Sun angle range, specified in Table 1. The Sun angle $\phi$, measured from the starshade normal, is shown in Figs. 1(a) and 4(b). For HabEx, with a single continuous band, the coating reflectance is averaged from 300 to $1000 \mathrm{~nm}$ and $40 \mathrm{deg}<\phi<85 \mathrm{deg}$. For SRM, with two bands operated at different distances, the average is first performed individually over the blue and green bands, and the two bands are then weighted by the inverse square of their operating distance. Thus, the green band is weighted by a factor of 2.1 relative to the blue band.

The reflectances calculated on a flat surface serve as a heuristic for coating performance on the curved terminal edge. Generally, the darker the coating is, the better the overall suppression of edge-scattered light is. But, as will be shown, a significant performance improvement is seen when the layer thicknesses are adjusted to compensate for the combined diffraction and reflection at the curved terminal edge.

The scatter values in Table 2 represent the average of the incident intensity of the combined sand p- polarized states, per meter of edge, scaled to an observation distance of $1 \mathrm{~m}$, and weighted across the bands, for a specular edge orientation. To calculate the apparent magnitude of a $1 \mathrm{~m}$ long specular edge as it would appear to the Roman Space Telescope, we use a representative edge fractional intensity of $2 \times 10^{-9}$, a distance of $26 \mathrm{Mm}$, and account for the angular diameter of the Sun which spreads the light by 0.01 radians along the length of the edge, resulting in an observed fractional intensity of $2 \times 10^{-9} / 0.01 /\left(26 \times 10^{6}\right)^{2}=3 \times 10^{-22}$ of the incident sunlight on the edge. This is an attenuation of 53.8 magnitudes which given the Sun's visual magnitude of -26.7 would lead to an integrated magnitude for the edge of $V=27.1$.

\subsection{Manufacturing Approach}

ZeCoat's standard motion-controlled coating system makes flat coatings by moving an evaporation source down the radius of the coating area, while the substrate is rotated above the source. To coat the edge of a blade, which can be considered a two-dimensional surface, the approach was to move the source linearly past the substrate, rather than rotating the substrate, as shown in Fig. 3.

A quartz-crystal microbalance tracks the amount of mass applied to the substrate as a function of position on the coated substrate. The translation speed is adjusted with a closed-loop feedback system based on changes in coating flux leaving the evaporation source. Noise from 
Table 2 Coating characteristics and results for the assortment of coatings designed and evaluated. Reflectance and scatter results are averaged over their respective mission bands and simulated using both the LoS model and the uniform coating model, with the best performing member of each column emphasized in bold. The reflectance and scatter results are also presented for an uncoated AM edge, providing a starting point for each coating.

\begin{tabular}{|c|c|c|c|c|c|c|c|}
\hline \multirow[b]{3}{*}{ Coating design } & \multirow{3}{*}{$\begin{array}{c}T \\
(\mathrm{~nm})^{\mathrm{a}}\end{array}$} & \multicolumn{3}{|c|}{ Average for $\mathrm{SRM}^{\mathrm{b}}$} & \multicolumn{3}{|c|}{ Average for $\mathrm{HabEx}^{\mathrm{c}}$} \\
\hline & & \multirow{2}{*}{$\begin{array}{c}\text { Reflection } \\
(\%)\end{array}$} & \multicolumn{2}{|c|}{ Scatter ${ }^{*} 1 e 9^{d}$} & \multirow{2}{*}{$\begin{array}{l}\text { Reflection } \\
(\%)\end{array}$} & \multicolumn{2}{|c|}{ Scatter ${ }^{*} 1 e 9^{d}$} \\
\hline & & & LoS & Uniform & & LoS & Uniform \\
\hline Uncoated AM substrate & 0 & 54.56 & 29.14 & & 53.65 & 35.64 & \\
\hline $400: 1000-1$ & 350 & 3.02 & 2.18 & 7.44 & 10.52 & 6.71 & 17.37 \\
\hline $400: 1000-2$ & 259 & 3.09 & 3.27 & 5.23 & 7.91 & 5.81 & 12.37 \\
\hline $400: 1000-3$ & 368 & 4.68 & 3.09 & 8.71 & 11.68 & 7.18 & 19.05 \\
\hline $400: 1000-4$ & 311 & 11.16 & 5.57 & 15.74 & 17.82 & 10.55 & 27.07 \\
\hline $400: 750$ & 200 & 2.95 & 1.75 & 4.37 & 8.36 & 6.17 & 10.81 \\
\hline $450: 1000-1^{e}$ & 301 & 4.49 & 1.96 & 7.18 & 7.71 & 5.06 & 14.06 \\
\hline $450: 1000-2$ & 131 & 4.49 & 5.40 & 5.51 & 11.08 & 11.20 & 12.68 \\
\hline $450: 1000-3$ & 256 & 4.63 & 2.30 & 6.55 & 7.82 & 5.09 & 12.80 \\
\hline $450: 1000-4$ & 70 & 8.57 & 5.57 & 8.58 & 17.81 & 16.72 & 19.23 \\
\hline $450: 1000-57.5^{\circ}-1$ & 233 & 5.19 & 5.38 & 8.88 & 9.53 & 8.05 & 15.37 \\
\hline $450: 1000-57.5^{\circ}-2^{\mathrm{e}}$ & 244 & 7.10 & 9.42 & 10.46 & 10.56 & 11.85 & 16.36 \\
\hline $450: 1000-57.5^{\circ}-3$ & 204 & 7.34 & 10.54 & 9.19 & 10.87 & 12.74 & 14.71 \\
\hline $450: 800-1$ & 341 & 2.95 & 1.25 & 6.49 & 8.90 & 5.87 & 16.09 \\
\hline $450: 800-2^{e}$ & 175 & 3.68 & 3.25 & 4.15 & 7.50 & 7.06 & 9.04 \\
\hline $450: 800-3^{e}$ & 220 & 3.81 & 2.95 & 5.09 & 7.68 & 7.19 & 10.84 \\
\hline $450: 800-60^{\circ}-1$ & 306 & 3.34 & 2.75 & 6.80 & 7.52 & 4.93 & 13.93 \\
\hline $450: 800-60^{\circ}-2$ & 265 & 4.69 & 5.12 & 4.72 & 7.34 & 7.63 & 11.14 \\
\hline $450: 900$ & 207 & 3.53 & 4.07 & 4.75 & 7.87 & 6.98 & 9.85 \\
\hline $600: 700$ & 78 & 9.93 & 6.92 & 8.75 & 20.77 & 19.86 & 20.92 \\
\hline $650-45^{\circ}-1$ & 191 & 3.39 & 1.79 & 4.35 & 8.34 & 6.11 & 10.48 \\
\hline $650-45^{\circ}-2$ & 268 & 3.49 & 1.68 & 5.88 & 8.46 & 6.24 & 13.42 \\
\hline
\end{tabular}

${ }^{\mathrm{a} C o a t i n g ~ t h i c k n e s s ~ i n ~ n a n o m e t e r s . ~}$

${ }^{\text {b} R e f l e c t a n c e ~ a n d ~ s c a t t e r ~ m e a s u r e m e n t s ~ a v e r a g e d ~ o v e r ~ S R M ~ S u n ~ a n g l e s ~ a n d ~ b o t h ~ b a n d s, ~ w i t h ~ t h e ~ b l u e: g r e e n ~}$ bands weighted 1:2.1 due to the green band's closer range.

${ }^{\mathrm{C}}$ Reflectance and scatter measurements averaged over the HabEx band and Sun angles.

dScatter units are the fraction of light intensity incident on the edge per meter of edge that appears at the location of the telescope aperture at an observation distance of $1 \mathrm{~m}$. The fraction scales linearly with the illuminated edge length and inversely as the square of the distance to the edge. The fraction is averaged over the relevant bandpass and solar angle range.

'Manufactured and tested design. 


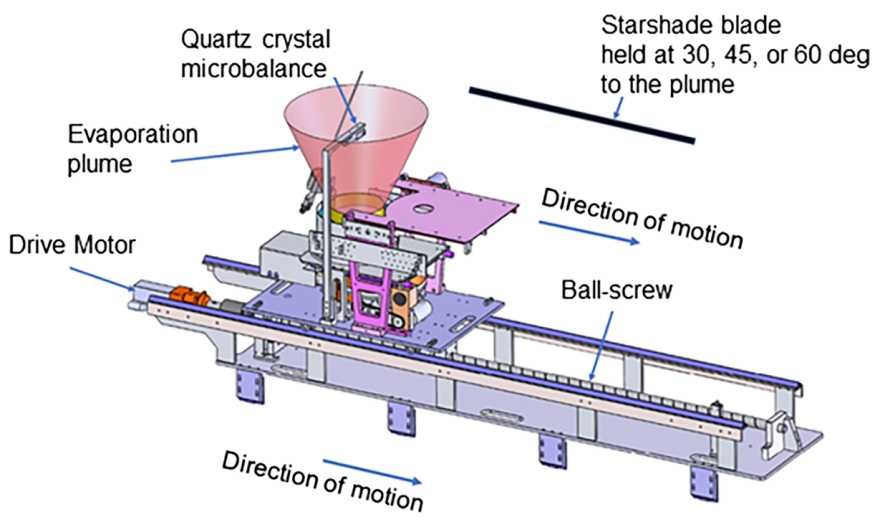

Fig. 3 Motion-controlled coating system employed at ZeCoat, Inc.

the crystal sensor is filtered with software. The entire coating plume is moved completely past the blade (to account for plume nonuniformities) so that all points on the blade's edge receive the same volume of coating. Edges were held at angles, $\alpha$, between $30 \mathrm{deg}$ and $60 \mathrm{deg}$, for this study. Mass tracking (as opposed to the industry standard, "optical monitoring," which uses interference to determine coating thickness) is particularly useful for precisely coating very large substrates greater than $1 \mathrm{~m}$ in diameter or length. This process achieves a coating uniformity of $\pm 2 \%$ over 2-m diameters.

\subsection{Environmental Tests}

We performed tape adhesion, abrasion, 24-h humidity, and thermal cycling tests to evaluate robustness on coating $450: 1000-57.5^{\circ}-2$. This coating is representative of the others with the same manufacturing process and materials, with some differences in the number of layers and thicknesses. The coating was tested for adhesion to the substrate by applying $3 \mathrm{M}$ Magic $810^{\mathrm{TM}}$ tape to the surface and slowly removing the tape by pulling at $90 \mathrm{deg}$. The coating tightly adhered to the AM foil surface and showed no loss of adhesion.

The smooth coatings are also hard and durable. The coating was tested per the Mil-PRF13830B paragraph B.4.4.5 moderate abrasion test and showed no damage or particulate contamination.

The coating passed a 24-h humidity test per Mil-PRF-13830B paragraph B.4.4.7, which calls for $>95 \%$ humidity and a temperature of $120 \pm 4^{\circ} \mathrm{F}$. The certified testing was performed at Environmental Associates, Inc., California.

Thermal cycling tests were performed at ZeCoat and Environmental Associates, Inc. In ZeCoat's test, we rapidly heated a coated sample by placing it in a preheated oven at $170^{\circ} \mathrm{C}$ for $1 \mathrm{~h}$. The sample was then removed and cooled on the lab bench back to room temperature. The coating was tape-tested after cooling, with standard cellophane tape and a very aggressive tape that far exceeds forces generated with cellophane tape, and showed no loss of adhesion and no physical changes. The same coated foil sample was then wrapped in bubble wrap with a thermocouple, and both were heat-sealed into a plastic bag. The bag was placed in an insulated box (a cooler) filled with frozen carbon dioxide and rapidly cooled to $-75^{\circ} \mathrm{C}$. Afterward, the sample was removed, warmed to room temperature, and tape-tested with aggressive tape. The coating showed no loss of adhesion and no physical changes.

A second, relatively benign certified test, comprising three cycles from $-20^{\circ} \mathrm{C}$ to $+50^{\circ} \mathrm{C}$, was administered at Environmental Associates, Inc. and resulted in no apparent damage.

\section{Model Description}

We modeled both an LoS coating, typical of the physical vapor deposition used here, as well as a uniform coating that could be achieved by rotating the part during coating or with a chemical vapor deposition process. These are illustrated in Fig. 4. The LoS coating presents less surface 


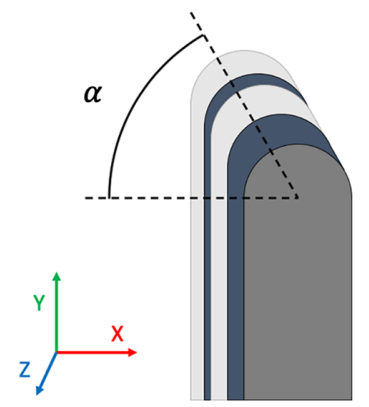

(a)

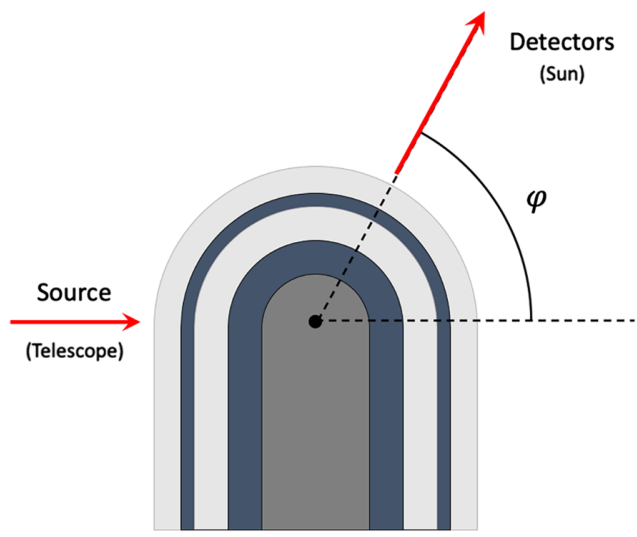

(b)

Fig. 4 Geometry of the two coating models: (a) the LoS model, coated at angle $\alpha$, and (b) the uniform coating model, with the incident and scattered light directions.

area but leaves one side with little to no coating material. The uniform coating is more akin to an AR surface coating in which the light enters and exits through the coating after reflecting from the substrate.

We employed the Lumerical FDTD suite to model the scatter of coated edges. Figure 5 shows the overall setup of the simulation. The two-dimensional simulation takes place in the $x y$-plane and considers the cross-section of the coated edge. A linearly polarized, broadband Gaussian beam is normally incident on the edge, which scatters light into field monitors located on a circular ring $15 \mu \mathrm{m}$ away from the center of curvature of the edge. The beam is incident from the left, and the scatter is analyzed on the right over a range of angles $-85 \mathrm{deg}<\phi<85 \mathrm{deg}$. This mimics the geometry of the multiangle scatterometer (MAS) at JPL ${ }^{14}$ which is the reverse of the geometry of the starshade in space.

On the starshade, we also distinguish between the half of the optical edge that can directly reflect sunlight into the telescope, which we refer to as the leading edge, with $\phi>0 \mathrm{deg}$, and the other half that cannot directly reflect, dubbed the trailing edge, with $\phi<0$ deg. In Lumerical, both the leading and trailing edges can be analyzed with a single simulation. While only the leading edge reflects light, the diffraction is present for both the leading and trailing edges. The trailing edge scatter of coated edges was analyzed to ensure that the coating does not simply

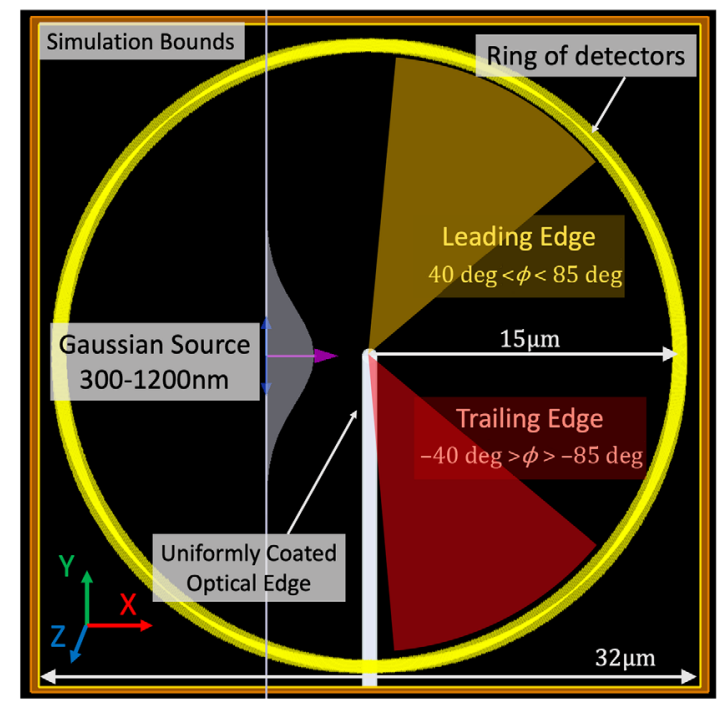

Fig. 5 Lumerical simulation region overview. 
redirect light from the leading edge region into the trailing edge region. While we found a slight enhancement, the scatter was still well below the level of the leading edge and did not significantly degrade performance. This will be the subject of a future paper.

During the edge simulation, the top half of the incident Gaussian beam is largely unaffected by the edge and passes through to the field monitors, where it interferes with the scattered beam. This will not happen in space, where the telescope is thousands of kilometers from the starshade and the scattered sunlight is incoherent with direct sunlight at the telescope. When analyzing the leading edge scatter, this incident field is removed by running a preliminary simulation with no edge in place, recording the incident electric field, and then subtracting it from the total field. When analyzing the trailing edge, the edge blocks the incident pulse from entering the field monitors, so the preliminary simulation and subtraction are not required.

\subsection{LoS Model}

The AM substrate is constructed using a $150-\mathrm{nm}$ radius circle as the terminal edge and a $300-$ $\mathrm{nm}$ wide rectangle making up the body of the substrate. Each coating layer is then added to the substrate by copying the substrate geometry and shifting it by the layer thickness in the direction of the coating LoS. Where the coating is curved along the top edge, it is necessary to add a rectangle, rotated by $\alpha$, to smoothly bridge from the current layer to the underlying layer, imitating the process of building up the coating along the LoS. The outermost layer with the highest mesh order is created first, then the next layer inward is added, overlapping and overwriting the material within, followed by each subsequent layer, and ending with the substrate core.

\subsection{Uniform Model}

This model consists of a nested set of circular edges with rectangular bodies with widths that match the diameters of the edges. The circular edges are concentric at the origin with bodies that extend down to the bottom of the simulation region.

\subsection{Boundary Conditions}

The simulation region consists of a $32 \times 32 \mu \mathrm{m}^{2}$ area using perfectly matched layers (PML) at each boundary. This size is determined by the detector range (Sec. 3.6), allowing the scatter to be analyzed at any angle. The PML begins at the outside edge of the simulation region and uses the steep angle profile with 12 layers, ensuring that obliquely scattered light is sufficiently absorbed at the boundary and does not cause extraneous reflections. Other settings, such as the parameters that fine-tune the PML properties, were left at their default values.

\subsection{Mesh Settings}

Unless otherwise noted, all results were simulated using an auto-nonuniform mesh with a mesh accuracy setting of one, corresponding to a minimum sampling of six points/wavelength. To capture the finer detail present in the layered edge, a mesh override region that provided a finer uniform mesh spacing of $2 \mathrm{~nm}$ was placed around the tip. To reduce simulation run time, this tip mesh extended only past the outermost layer, rounded to the nearest tenth micron. The autononuniform mesh increases the sampling near the border of the mesh override region. This serves to smooth out the transition from the relatively low-resolution background mesh to the highresolution region around the edge tip.

A convergence test with varying tip mesh spacings was performed on coating 450:800-2 to verify the accuracy of the simulation results. We compared the scatter averaged over SRM bands and solar angles and summarize the results in Table 3. The scatter results are normalized to the highest accuracy setting of $0.5 \mathrm{~nm}$. These results show that scatter results obtained using a 2-nm mesh differ by $1.3 \%$ from the highest accuracy setting. Results reported below use a $2-n m$ mesh unless otherwise stated. 
Table 3 Convergence test results.

\begin{tabular}{ccccc}
\hline \hline \multicolumn{2}{c}{ Tip mesh convergence } & & \multicolumn{2}{c}{ Detector range convergence } \\
\cline { 5 - 6 } $\begin{array}{c}\text { Tip mesh } \\
\text { spacing } \\
(\mathrm{nm})\end{array}$ & $\begin{array}{c}\text { Normalized } \\
\text { average SRM } \\
\text { scatter }\end{array}$ & & $\begin{array}{c}\text { Detector } \\
\text { range } \\
(\mu \mathrm{m})\end{array}$ & $\begin{array}{c}\text { Normalized } \\
\text { average SRM } \\
\text { scatter }\end{array}$ \\
\hline 0.5 & 1 & & 2 & 1.0650 \\
1 & 0.994 & & 5 & 1.0219 \\
2 & 0.987 & & 10 & 1.0074 \\
3 & 0.981 & & 15 & 1.0051 \\
5 & 0.961 & 20 & 1 \\
\hline \hline
\end{tabular}

\subsection{Source Settings}

The source consists of a Gaussian beam with a 5- $\mu \mathrm{m}$ waist radius. The bandpass spans the wavelengths 300 to $1200 \mathrm{~nm}$. The beam is generated using the scalar approximation setting and is placed $5 \mu \mathrm{m}$ away from the edge, with the beam waist focused on the origin, coinciding with the edge's center of curvature. The 5- $\mu \mathrm{m}$ waist radius is wide enough to ensure that the tip of the edge receives spatially uniform incident light and that the beam does not significantly diverge between the edge tip and the detectors, while also being narrow enough to allow the source to taper off sufficiently before encountering the top and bottom edges of the simulation region, preventing stray reflections from the PML region.

The polarization angle of the source is set $45 \mathrm{deg}$ to the edge in the $y z$-plane to generate equal light in the s- and p-polarizations. In this configuration, each polarization can be extracted and analyzed separately by examining the separate $x-/ y$-, or $z$-components of the scattered field. The $z$-component of the electric field makes up the s-polarized light, and the $x$ - and $y$-components make up the p-polarized light. Examining the separate polarizations allows us to analyze the polarization-dependent effects of different designs.

\subsection{Detector Settings}

The detectors consist of field and power point monitors placed along a $15-\mu \mathrm{m}$ radius ring centered on the origin and spaced evenly every quarter degree. The detectors span the full $360 \mathrm{deg}$ around the edge. The detectors collect the field profile in the frequency domain and report it at 19 points spaced every $50 \mathrm{~nm}$, spanning the 300 - to $1200-\mathrm{nm}$ wavelength range. Collecting the field, rather than the intensity, allows for the incident pulse to be coherently subtracted from the total scattered field.

The detectors must be placed far enough away to measure the far-field behavior, but close enough to limit simulation run times to a reasonable level. Table 3 summarizes the results of a convergence test comparing the average scattered signal at a range of distances from a $150 \mathrm{~nm}$ radius uncoated AM edge. Each result was scaled linearly to $20 \mu \mathrm{m}$ and normalized to the scatter at that distance. Table 3 indicates that the signal stabilizes at a distance of $\sim 10 \mu \mathrm{m}$ and changes by just $0.5 \%$ beyond $15 \mu \mathrm{m}$. All results presented in this paper were computed at a range of $15 \mu \mathrm{m}$, where run times were reasonable, typically $\sim 10$ minutes per coating on a modern laptop computer.

\section{Coating Performance}

\subsection{Comparison with Experimental Data}

Preliminary scatter data were gathered experimentally using the single-angle scatterometer (SAS) at JPL. Details of the design and calibration of the SAS are given in Shaklan et al., ${ }^{9}$ with a brief description given here. Figure 6 shows the setup of the instrument. A linearly polarized 


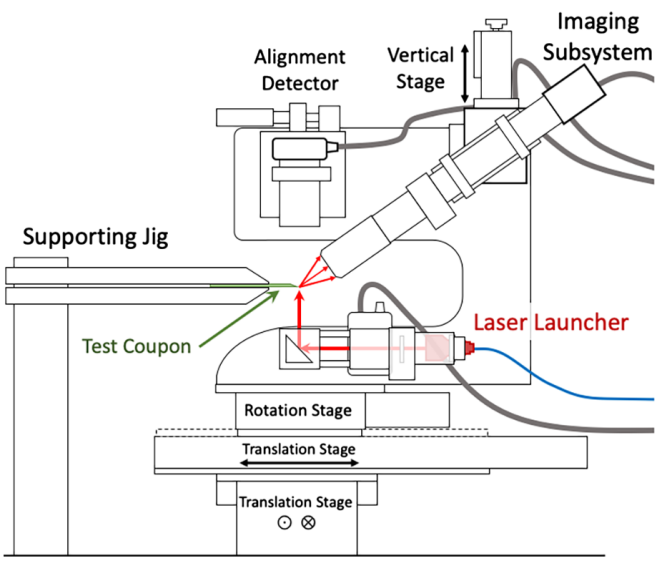

(a)

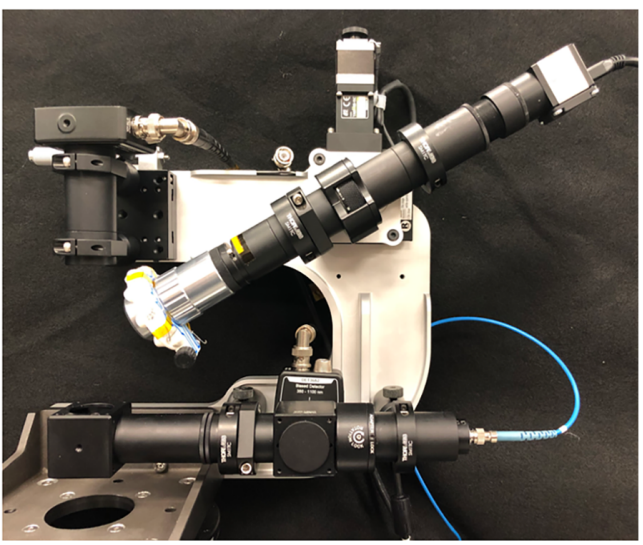

(b)

Fig. 6 (a) Schematic of the single-angle scatterometer and (b) picture of the assembled instrument.

laser illuminates the edge of a test coupon from below and scatters light into a high-resolution camera. The camera gathers s-polarized, $635-\mathrm{nm}$ light with a long working distance $10 \times$ microscope objective with a $30 \mathrm{deg}$ acceptance angle that is held at $60 \mathrm{deg}$ relative to the plane of the test coupon. The instrument forms an image of a 1-mm length of the edge on the camera. The instrument software centers and refocuses the camera on the edge at each point before taking an image. The pixel values in the resulting image are summed and normalized by the exposure time and the incident laser power. To determine the absolute level of scatter, the data are compared with a reference coupon with an absolute scatter ratio that was measured with another instrument, the MAS. ${ }^{9}$ The SAS then moves along the length of the test coupon to the next point and repeats the alignment and measurement process, eventually measuring the scatter performance of the test coupon along its entire length.

Figure 7 shows the results for four manufactured coatings, each applied to three coupons at different coating angles, as well as a representative uncoated coupon, labeled B27 for its manufacturing lot and sequence. Table 4 summarizes the median performance of each edge. Both Fig. 7 and Table 4 include the average value of the median performance of 13 representative uncoated coupons comanufactured with the ones presented here. We also show the Sommerfeld diffraction limit ${ }^{12}$ for a semi-infinite, perfectly conducting, infinitesimally thick half-plane. The uncoated edges scatter light at close to this value, while the coated edges scatter much less light. The measurement errors in Table 4 include the quadrature addition of a $1 \sigma$ value of $\pm 16 \%$ determined from instrument calibration and coupon statistics reported in our previous work ${ }^{9}$ and a $\pm 30 \%$ estimate of the standard error of the median. The standard error of the median was determined by measuring a 500-mm long -coated edge (Fig. 8) and computing the standard deviation

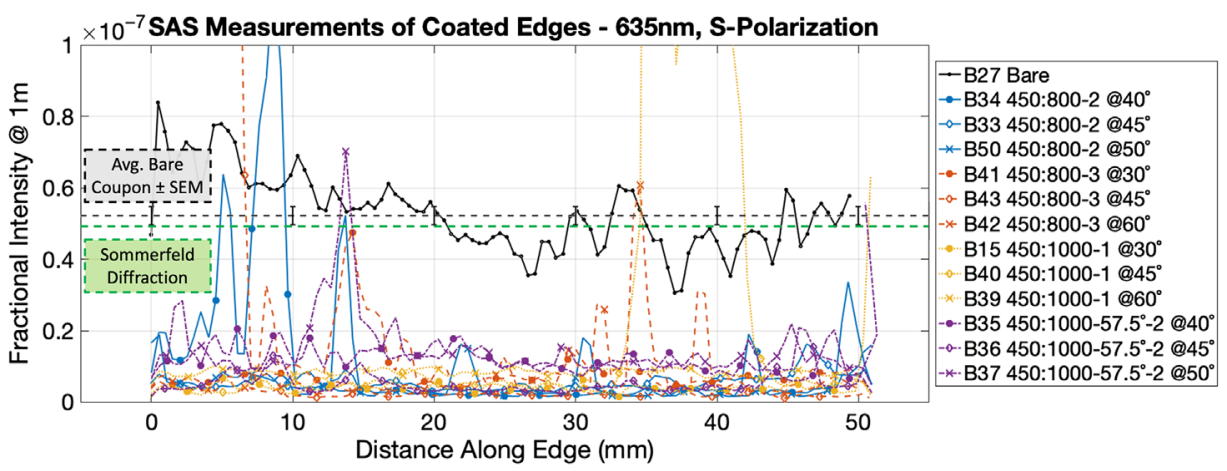

Fig. 7 SAS measurements of coated coupons and a representative uncoated coupon serving as a reference point. The average performance of 13 coupons, as well as the value for Sommerfeld half-plane diffraction, is marked. 
Table 4 Summary of SAS measurements (s-polarization) of preliminary coated edges as well as a representative uncoated edge, which serves as a baseline to gauge performance improvement.

\begin{tabular}{lccccc}
\hline \hline & & & Coating angle \\
Sample name & Date & Coating & $\begin{array}{c}\text { Median } \\
\text { scatter }{ }^{*} \text { e9 }\end{array}$ & $\begin{array}{c}\text { Error } \\
(1-\sigma)\end{array}$ \\
\hline B34 & October 26, 2020 & $450: 800-2$ & 40 & 2.89 & 0.98 \\
B33 & October 26, 2020 & $450: 800-2$ & 45 & 4.54 & 1.54 \\
B50 & October 26, 2020 & $450: 800-2$ & 50 & 2.98 & 1.01 \\
B41 & February 5, 2020 & $450: 800-3$ & 30 & 7.32 & 2.48 \\
B43 & February 5, 2020 & $450: 800-3$ & 45 & 2.09 & 0.71 \\
B42 & February 5, 2020 & $450: 800-3$ & 60 & 4.27 & 1.45 \\
B15 & February 13, 2020 & $450: 1000-1$ & 30 & 3.97 & 1.35 \\
B40 & February 13, 2020 & $450: 1000-1$ & 45 & 5.19 & 1.76 \\
B39 & February 13, 2020 & $450: 1000-1$ & 60 & 8.44 & 2.86 \\
B35 & October 22, 2020 & $450: 1000-57.5^{\circ}-2$ & 40 & 11.52 & 3.91 \\
B36 & October 22, 2020 & $450: 1000-57.5^{\circ}-2$ & 45 & 4.16 & 1.41 \\
B37 & October 22, 2020 & $450: 1000-57.5^{\circ}-2$ & 50 & 11.72 & 3.98 \\
B27 & October 22, 2019 & Uncoated & N/A & 52.88 & 17.95 \\
Uncoated average & Various & Uncoated & N/A & 52.31 & $2.60($ SEM) \\
\hline \hline
\end{tabular}

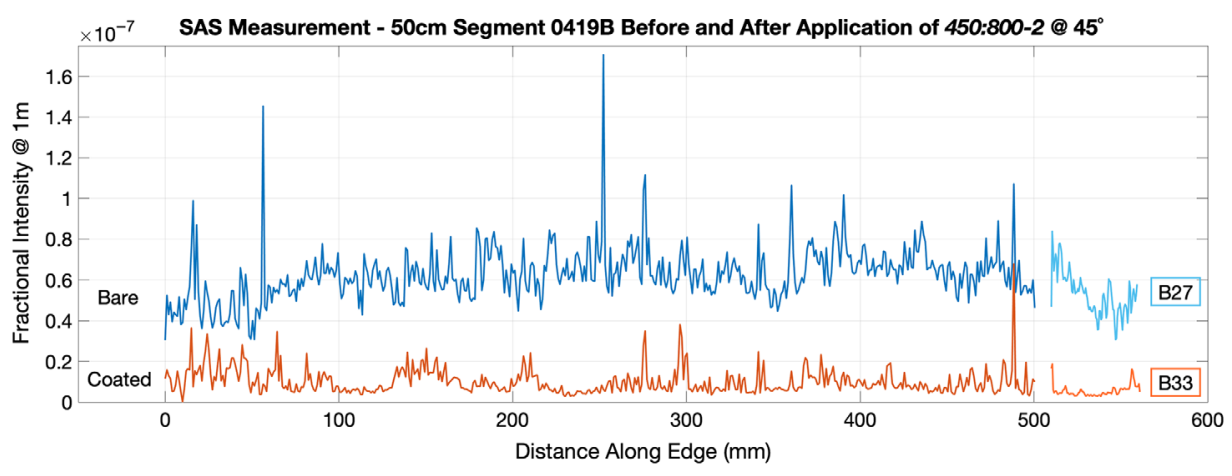

Fig. 8 Before and after SAS measurements of a $50 \mathrm{~cm}$ AM edge segment that was coated with 450:800-2 at an angle of $45 \mathrm{deg}$. Also included in this plot is the representative uncoated coupon B27, as well as the coupon B33, which was coated in the same manner as the segment.

of the median of 10 adjacent 50-mm long subsamples, each representing a potential coupon. The final estimated measurement error for the individual coupons was then $34 \%$.

The variability of the scatter along the 50-mm length of coupon B27 is typical of all uncoated coupons that we tested. The relative variability in the coated edges is higher. From the SAS images, each of which covers $1 \mathrm{~mm}$ of length along the edge, we find that the bright spots, visible in Fig. 7, on coupons B43 (0 to $10 \mathrm{~mm}$ ) and B40 (33 to $43 \mathrm{~mm}$ ) are both from physical damage to the substrate. The root cause of this damage is unknown, although it may have happened during installation in the mount. Other defects such as those on coupon B41 (13 to $18 \mathrm{~mm}$ ) and coupon B42 (30 to $40 \mathrm{~mm}$ ) do not exhibit obvious signs of damage. Instead, these are likely to be the result of dust or fibers landing on the edge prior to coating when the edges were handled in standard laboratory environments. Since performing these tests, the coating chamber has been 


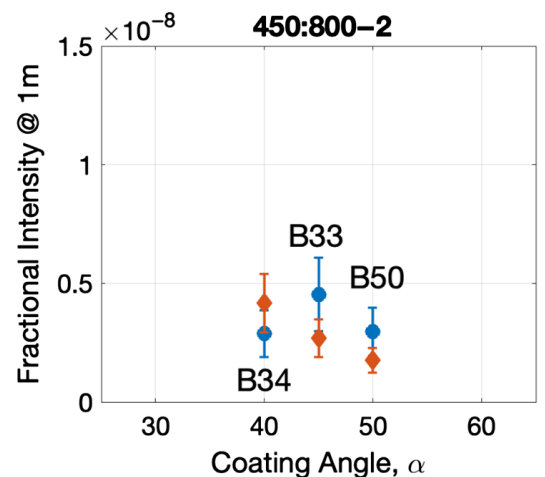

(a)

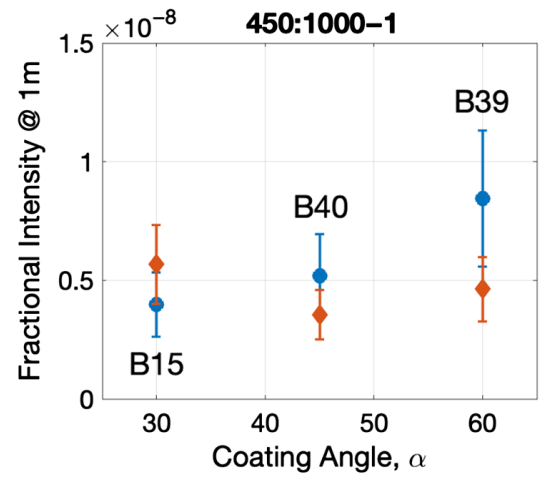

(c)

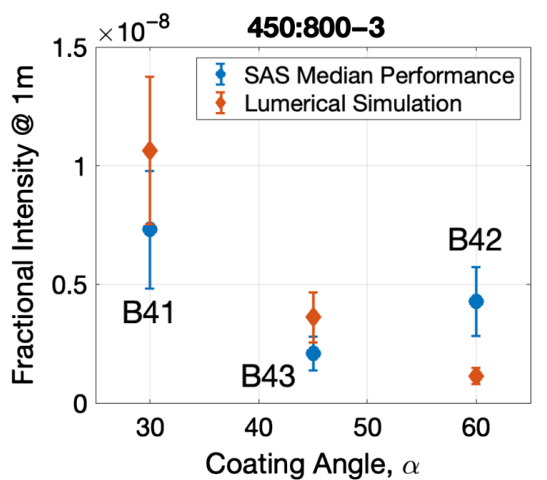

(b)

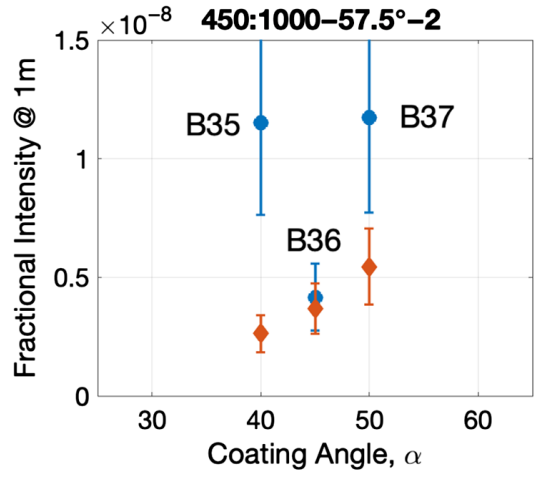

(d)

Fig. 9 Comparison between the s-polarized SAS experimental data and Lumerical results for a wavelength of $635 \mathrm{~nm}$ for coatings (a) 450:800-2, (b) 450:800-3, (c) 450:1000-1, and (d) 450:1000$57.5^{\circ}-2$. Experimental error bars are $\pm 34 \%$, and model error bars are $\pm 29 \%$ as per Sec. 4.1 .

relocated to a new cleanroom and we have developed new safe handling procedures, which should reduce the number of defects and improve scatter performance along the coupon edges and, ultimately, the entire starshade optical edge.

Along with the 12 coated 50-mm coupons, we also measured a 50-cm AM edge segment before and after coating with 450:800-2 at $45 \mathrm{deg}$ (Fig. 8). The results for coupons B27 (uncoated) and B33 (450:800-2 @ 45 deg) are also plotted for reference. The coating reduced measured scatter by nearly a factor of $8 \times$ on average with a range of $1.5 \times$ to $20 \times$. There are several scatter features present in the uncoated scatter measurement that are still identifiable in the coated segment. However, the two largest spikes at 56 and $252 \mathrm{~mm}$ cannot be seen postcoating. These spikes are most likely due to debris on the segment, which was cleaned prior to coating.

We compare our experimental results with the model results in Fig. 9. These results validate the overall accuracy of the models and suggest that coating formulations and application angles could be optimized to further reduce scatter. Model error bars include the error associated with substrate radius uncertainty as well as the error associated with manufacturing tolerances and refractive index uncertainties. As evidenced in Fig. 2, the terminal edge radius of the AM substrate varies along its length; we estimate that the radius ranges between 100 and $200 \mathrm{~nm}$, with a mean value of $150 \mathrm{~nm}$. We used Lumerical to simulate a coating over this range of edge radii and found that the scatter changed by $\pm 25 \%$.

The individual coating layers are manufactured to a tolerance that is specific to the material being evaporated. All materials used here can currently be manufactured to within $\pm 1.5 \mathrm{~nm} 1 \sigma$. Errors in the refractive index data of 3\% (also material-dependent, measured by ellipsometry on several sample coatings) are included as a first-order effect on the layer thicknesses. To evaluate the effect of refractive index and layer thickness errors on the scatter, we evaluated a set of 25 simulated coatings in a Monte-Carlo analysis, resulting in a $\pm 15 \%$ scatter standard deviation. 


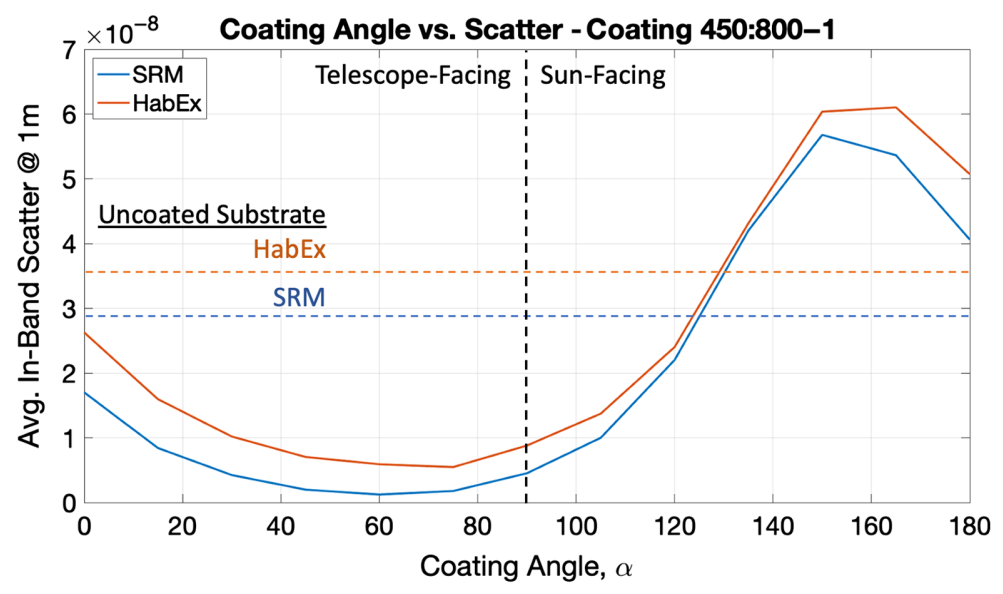

Fig. 10 Plot of the average in-band scatter as a function of coating angle. Note the minimum around $\alpha=60 \mathrm{deg}$ and the maximum near $\alpha=150 \mathrm{deg}$. The average in-band scatter from an uncoated substrate is also marked for each mission.

Combining in quadrature the scatter deviation due to index and thickness errors with the deviation due to radius uncertainty, we find total model uncertainty of $\pm 29 \%$. These error bars are plotted in Fig. 9.

\subsection{LoS Coating Study}

We studied the effect of coating at different angles using coating 450:800-1, which had the best performance in our initial study. Figure 10 shows the scatter results for both SRM and HabEx. A shallow minimum is achieved near $\alpha=60 \mathrm{deg}$. This minimum roughly coincides with the half-angle between the incident light direction and the scattered light direction, $40 \mathrm{deg}<\phi<85 \mathrm{deg}$. The scatter is maximized at $\alpha \sim 150 \mathrm{deg}$, with the coating on the sunlit side of the edge. At this angle, the sunlight reflects directly from the coating into the telescope, and the edge glint is brighter than the uncoated substrate. From these results, a coating angle of $60 \mathrm{deg}$ was chosen to analyze the set of coatings presented in Table 2.

Compared with the scatter from an uncoated 150 -nm radius AM substrate, all coating formulations provide a significant reduction, with the best result for SRM coming from 450:800-1, which reduced edge scatter by a factor of 25 . The best result for the HabEx mission was 450:800$60^{\circ}-1$, reducing the edge scatter by a factor of 8 .

The relationship between reflectance and scatter, as a function of wavelength and Sun angle, is shown in Fig. 11 for one particular coating, while the angle and wavelength-averaged reflectance is plotted for all of the coatings in Fig. 12. For the LoS coating, Fig. 11(b) shows a weak correlation between reflectance and scatter through the band and over the range of Sun angles.

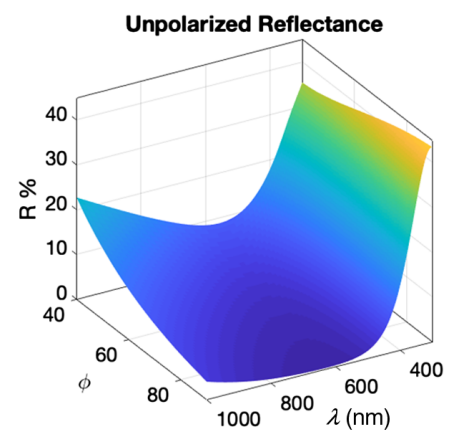

(a)

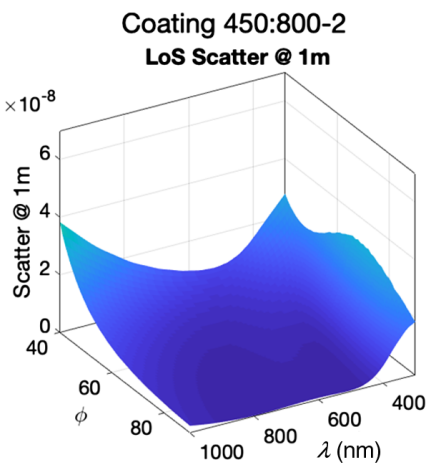

(b)

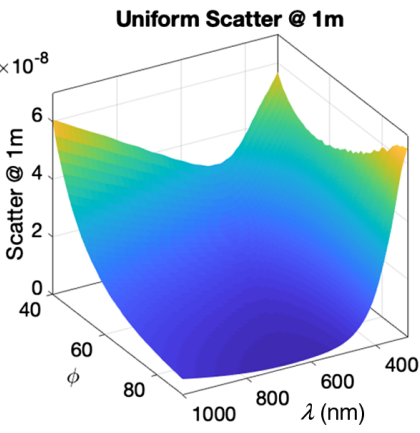

(c)

Fig. 11 Surface plots showing (a) the unpolarized reflectance, (b) the LoS coating scatter, and (c) the uniform coating scatter for coating 450:800-2. 


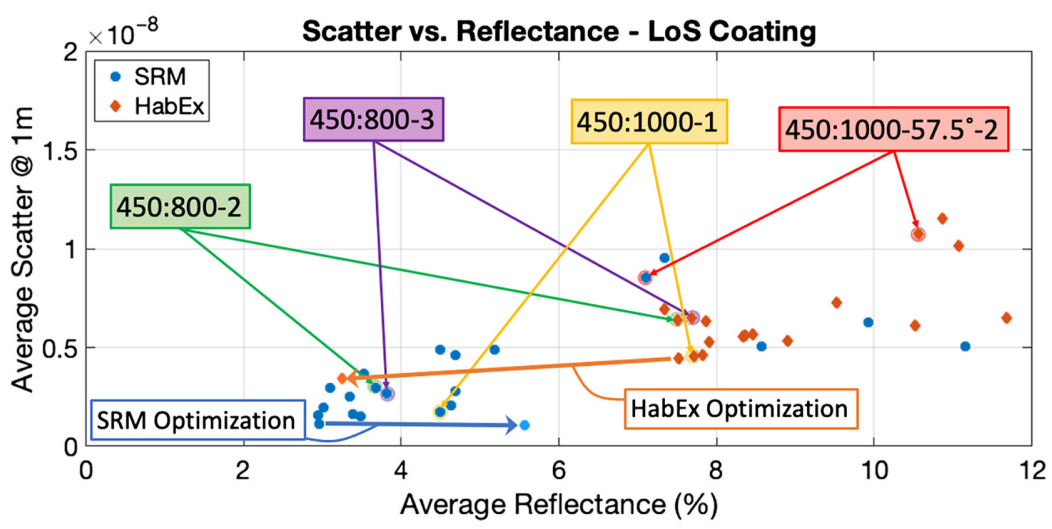

Fig. 12 Plot of the average reflectance versus scatter for the LoS model for both SRM and HabEx, showing a weak positive correlation between reflectance and scatter. Coatings that were manufactured and tested, as well as the starting and ending point for optimized SRM and HabEx designs, are labeled.

This is not surprising because the coating geometry differs strongly from the intended planar geometry.

Likewise, Fig. 12 shows a weak positive correlation between reflectance and scatter for all coatings. For the purpose of identifying design principles that would afford an efficient coating optimization and tolerancing approach, we considered different reflectance calculations that might better correlate with the scatter. We separately assessed shifting the angles of incidence to account for the coating angle, treating the coating as being thinned by $\cos \alpha$, and calculating the reflectance at normal incidence because the telescope is normal to the starshade surface. None of these variations, however, improved the correlation for the LoS coating.

\subsection{Uniform Coating Results}

We found that the correlation between reflectance and scatter over wavelength and Sun angle is strong for the uniform coating [Figs. 11(a) and 11(c)]. The correlation is worse at low angles, where the diffraction component is strongest, but for $\phi>50 \mathrm{deg}$, the correlation is clear. However, the correlation between average scatter and average reflectance for all coatings is weak. This is due to a key difference between the uniform and LoS coatings; the uniform coating surface area grows with coating thickness. When we plot the average scatter as a function of reflectivity and edge radius, where radius is the substrate radius plus the coating thickness, we find a clear linear relationship, with all of the scatter points falling on a plane (Fig. 13). The plane
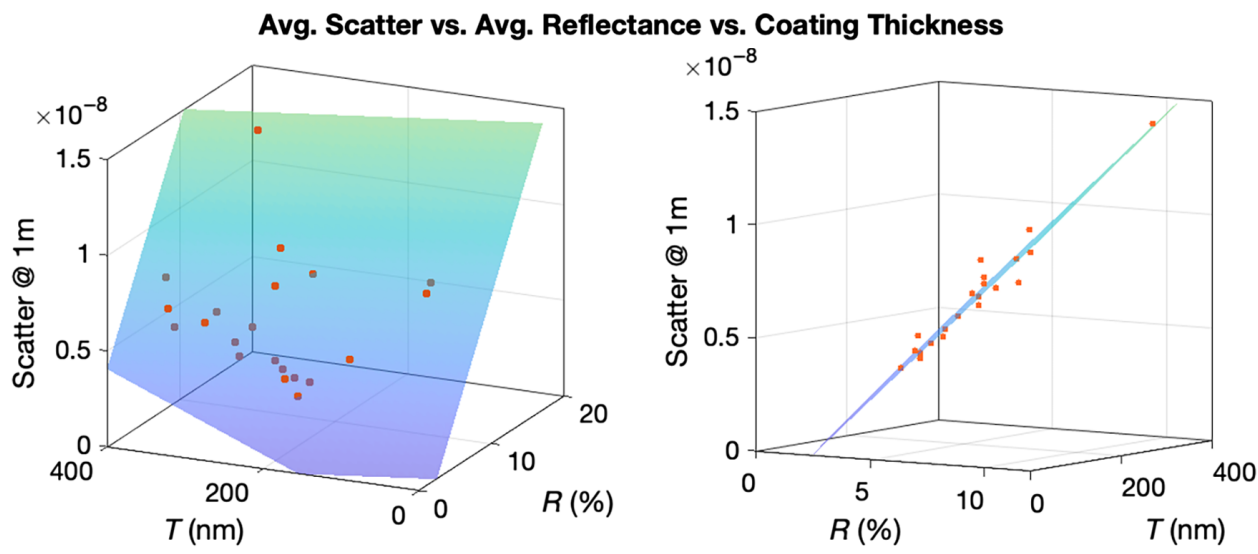

Fig. 13 Scatter plots of the uniform SRM scatter versus reflectance versus coating thickness for the coatings listed in Table 2. A planar fit is achieved describing the average scatter as the linear combination of average reflectance and coating thickness. 
is a good fit, with correlation coefficient $r^{2}=0.957$. A similar planar fit is also found for the HabEx band.

The linear dependence on thickness and reflectivity was surprising as we had expected the scatter to be a function of the product of the reflectance and radius. While this must be the case in the geometric regime, the results here show that the combination of subwavelength structures and significant levels of diffraction leads to a linear behavior for the uniform coated case. The plane indicates that low scatter can be obtained from a coating that is both very thin and has very low average reflectivity. Typically, in AR coating design, these two properties are at odds with one another, and it has proven difficult to design a coating that lies in this low-scatter regime. While we find a good planar fit to the simulated coatings, we do not expect to be able to populate the thin/low-reflectivity region.

\section{Design Optimization and Tolerancing}

The LoS scatter results in Table 2 demonstrate a significant improvement over the uncoated substrate. However, the purpose of these coating designs was exploratory in nature. To minimize the scattered light, and lacking a convenient proxy for the time-consuming FDTD calculation, our approach was to optimize the design using Lumerical's built-in particle swarm optimization utility. The coatings with the lowest scatter from Table 2 were chosen as starting points from which Lumerical adjusted the individual layer thicknesses with the objective of reducing the average scatter. Given the number of layers and layer materials, the utility runs many simulations to find the optimum layer thicknesses that minimize the average scatter for a given mission band. This is different from the initial coating design approach done by ZeCoat, which was based on minimizing the flat surface reflectance over different bands or angles of incidence. As seen in Fig. 12, the reflectance is only loosely correlated with overall edge scatter. The Lumerical software provides the capability of optimizing not just for surface reflectivity but for overall edge scatter. To facilitate this time-consuming set of simulations, we increased the tip mesh to 5-nm spacing. This decreased the accuracy of the result but did not change the relative performance of different coating designs. Once the optimization was finished, the final coating was reanalyzed with a high-resolution 2-nm tip mesh.

For SRM, the starting design was 450:800-1, and for HabEx, the starting design was 450:800-60 -1 . Table 5 presents the results of each optimization. For SRM, the optimization was able to reduce scatter by an additional $10 \%$. Averaged over the entire SRM band, this optimized coating reduces scatter by a factor of 28 compared with an uncoated edge. For HabEx, we started with $450: 800-60^{\circ}-1$ and found a design that further reduced scatter by $24 \%$. This optimized coating reduces the scatter from an uncoated edge by a factor of 10. Figure 12 shows the starting and ending points for SRM and HabEx LoS optimizations. The final designs had significantly different reflectivity than the starting points.

\subsection{Uniform Coating Optimization}

Optimization of the uniform coating designs took advantage of the linear dependence of scatter on coating thickness and reflectance. Using reflectance as a proxy for scatter, we optimized

Table 5 Initial and optimized results starting from the best coating for each coating model and mission. The last three columns are the initial, optimized, and toleranced scatter values.

\begin{tabular}{lccccccc}
\hline \hline $\begin{array}{l}\text { Coating } \\
\text { model }\end{array}$ & $\begin{array}{c}\text { Mission } \\
\text { band }\end{array}$ & $\begin{array}{c}\text { Initial } \\
\text { design }\end{array}$ & $\begin{array}{c}\text { Initial } \\
\mathrm{T}(\mathrm{nm})\end{array}$ & $\begin{array}{c}\text { Optimum } \\
\mathrm{T}(\mathrm{nm})\end{array}$ & $\begin{array}{c}\text { Initial } \\
\mathrm{S} * 10^{9}\end{array}$ & $\begin{array}{c}\text { Optimized } \\
\mathrm{S} * 10^{9}\end{array}$ & $\begin{array}{c}\text { Toleranced } \\
\mathrm{S} * 10^{9} \pm 1 \sigma\end{array}$ \\
\hline LoS & SRM & $450: 800-1$ & 341 & 358 & 1.25 & 1.13 & $1.17 \pm 0.04$ \\
& HabEx & $450: 800-60^{\circ}-1$ & 306 & 300 & 4.93 & 3.72 & $3.79 \pm 0.09$ \\
\multirow{2}{*}{ Uniform } & SRM & $450: 800-2$ & 175 & 181 & 4.15 & 3.58 & $3.64 \pm 0.08$ \\
& HabEx & $450: 800-2$ & 175 & 190 & 9.04 & 8.78 & $8.86 \pm 0.08$ \\
\hline \hline
\end{tabular}


scatter performance by adjusting the coating layer thicknesses using MatLab's standard "fminsearch" function while calling a vectorized version of the MatLab "jreftran" routine ${ }^{15}$ to efficiently evaluate reflectance. Once an optimized design was determined, it was evaluated in Lumerical to accurately measure the level of scatter.

Starting with 450:800-2 for both missions, the average scatter for SRM was reduced by $14 \%$. The improvement for HabEx was just $3 \%$.

\subsection{Manufacturing Tolerances}

The coating performance will be limited by manufacturing limitations. As mentioned in Sec. 2.3, the manufacturing process is capable of achieving a coating uniformity of $\pm 2 \%$ over a $2-\mathrm{m}$ length. The tolerance on individual layer thicknesses, as stated in Sec. 4.1, is currently $\pm 1.5 \mathrm{~nm}$, and in the future, with experience and refinements to the coating process, we expect to improve layer thickness tolerances to $\pm 1 \mathrm{~nm}$.

We performed Monte-Carlo simulations of the optimized coating designs to estimate the asmanufactured expected performance and its uncertainty, using the same process described in Sec. 4.1. Here, however, we do not account for refractive index uncertainty because the coating is assumed to be optimized for measured index data at the time of manufacture. For the LoS coating, which requires repeated Lumerical simulations, we ran 25 trials for each mission. For the uniform coating, the reflectivity-based scatter estimation function allowed a sample size of 10,000 trials. The performance of the toleranced coatings is presented in Table 5. All of the designs are expected to perform to within a few percent of the design point.

Table 6 Estimated glint lobe magnitude for optimized, asmanufactured coatings.

\begin{tabular}{|c|c|c|c|c|}
\hline $\begin{array}{l}\phi \\
\text { (deg) }\end{array}$ & $\begin{array}{l}\text { IWA phot. } \\
95 \% \text { conf. }\end{array}$ & $\begin{array}{l}\text { Improvement } \\
\text { ratio }\end{array}$ & $\Delta$ Mag & $\begin{array}{l}\text { Final } \\
\text { mag }\end{array}$ \\
\hline \multicolumn{5}{|c|}{ SRM 425 to $552 \mathrm{~nm}$ band } \\
\hline 53 & 27.3 & 9.2 & 2.4 & 29.7 \\
\hline 63 & 27.5 & 16.7 & 3.1 & 30.6 \\
\hline 73 & 27.3 & 26.8 & 3.6 & 30.9 \\
\hline 83 & 26.7 & 20.5 & 3.3 & 30.0 \\
\hline \multicolumn{5}{|c|}{ SRM 615 to $800 \mathrm{~nm}$ band } \\
\hline 53 & 25.2 & 14.3 & 2.9 & 28.1 \\
\hline 63 & 25.4 & 33.3 & 3.8 & 29.2 \\
\hline 73 & 25.2 & 61.0 & 4.5 & 29.7 \\
\hline 83 & 24.6 & 85.3 & 4.8 & 29.4 \\
\hline \multicolumn{5}{|c|}{ HabEx 300 to $1000 \mathrm{~nm}$ band } \\
\hline 35 & 27.2 & 4.0 & 1.5 & 28.7 \\
\hline 45 & 27.8 & 6.7 & 2.1 & 29.9 \\
\hline 55 & 28.1 & 11.2 & 2.6 & 30.7 \\
\hline 65 & 28.2 & 17.3 & 3.1 & 31.3 \\
\hline 75 & 27.9 & 23.1 & 3.4 & 31.3 \\
\hline 85 & 27.3 & 27.4 & 3.6 & 30.9 \\
\hline
\end{tabular}


We note that the toleranced, optimized coatings are only modestly better than the best measured coatings. For SRM, we measured coating 450:1000-1, which has a predicted scatter level of $1.96 \times 10^{-9}$ (Table 2), compared with the optimized coating 450:800-1 with a predicted scatter value of $1.17 \times 10^{-9}$, a $1.7 \times$ improvement. For HabEx, coating 450:1000-1 has a predicted scatter level of $5.06 \times 10^{-9}$ compared with the optimized coating $450: 800-60^{\circ}-1$ with a predicted performance of $3.79 \times 10^{-9}$, a $1.3 \times$ improvement. The laboratory measurements are consistent with performance that is within a factor of 1.7 of the best coating designs presented here.

\section{Magnitude of Solar Glint Lobes}

An estimate of the final solar glint magnitude is calculated based on the expected LoS coating improvement over an uncoated substrate. Table 6 reproduces the 95\% confidence level magnitude of the azimuthally averaged brightness of solar glint lobe within a diffraction-limited photometric aperture at the inner working angle (IWA) for uncoated AM edges published in Ref. 9. We show the improvement ratio for the expected as-manufactured performance (column 6 of Table 5) and equivalent $\Delta \mathrm{Mag}$ evaluated at several Sun angles. The rightmost column then shows the azimuthally averaged glint lobe magnitude at the IWA for coated edges.

For SRM, the weighting of the bands by the inverse of the starshade distance resulted in a $\sim 1$ magnitude closing of the performance gap between the blue and green bands. For both HabEx and SRM, the improvement factor is 2.1 magnitudes greater at the largest Sun angles compared with the smallest. A more uniform balance could be achieved, if desired, by weighting the Sun angles in the optimization.

\section{Conclusion}

We have measured the scatter from several thin AR coatings applied to the terminal edge of sharp metallic blades up to $50 \mathrm{~cm}$ long and have found that they reduce glint compared with uncoated edges by a factor of 5 to 20 . The tested coatings were designed using standard thin-film design software assuming a uniform coating on a flat plane; a further glint reduction factor of 1.7 can be gained when optimizing the designs to account for diffraction and edge curvature. When applied to the edges of starshade petals, an optimized coating can be expected to reduce solar glint, when averaged over the observing bandwidth and the range of solar angles, by a factor of $\sim 20$, to magnitude $\sim 29$ for SRM and $~ 30$ for HabEx. These estimates are based on FDTD models that have been validated by experimental results, and they include the expected performance with realistic manufacturing tolerances. A representative coating passed adhesion, humidity, hot and cold extremes, and thermal cycling with no apparent degradation.

In future work, we will manufacture and test the optimized designs. We will also expand our optimization approach to include a larger range of initial designs and a study of the initial coating angle, as well as weighting by the Sun angle to achieve a more balanced solution. Ultimately, the coatings will be tested to flight qualification and environmental levels.

\section{Acknowledgments}

The research was carried out at the Jet Propulsion Laboratory, California Institute of Technology, under a contract with the National Aeronautics and Space Administration (80NM0018D0004).

\section{References}

1. W. Cash, "Detection of earth-like planets around nearby stars using a petal-shaped occulter," Nature 442, 51-53 (2006).

2. S. Seager et al., "Starshade rendezvous probe mission," 2019, https://www.hou.usra.edu/ meetings/landscape2019/presentations/Seager.pdf (accessed 1 June 2020).

3. S. Seager et al., "The exo-s probe class starshade mission," Proc. SPIE 9605, 96050W (2015). 
4. A. Romero-Wolf et al., "Starshade rendezvous: exoplanet sensitivity and observing strategy," J. Astron. Telesc. Instrum. Syst. 7(2), 021210 (2021).

5. S. Gaudi et al., "Habitable Exoplanet Observatory," 2019, https://www.jpl.nasa.gov/habex/ (accessed 1 June 2020).

6. B. S. Gaudi et al., "The Habitable Exoplanet Observatory (HaBex)," Proc. SPIE 11115, 111150M (2019).

7. D. McKeithen et al., "Modeling the scatter of sunlight from starshade edges," Proc. SPIE 11117, 111171L (2019).

8. B. Crill and N. Siegler, "Exoplanet exploration program 2019 technology plan appendix," Jet Propulsion Laboratory Publications No. D-102506, 2019, https://exoplanets.nasa.gov/ exep/technology/technology-overview/ (accessed 1 June 2020).

9. S. Shaklan et al., "Solar glint from uncoated starshade optical edges," J. Astron. Telesc. Instrum. Syst. 7(2), 021204 (2021).

10. J. Steeves et al., "Development of low-scatter optical edges for starshades," Proc. SPIE 10706, 107065K (2018).

11. S. Casement et al., "Results of edge scatter testing for a starshade mission," Proc. SPIE 9904, 99043H (2016).

12. M. Born and E. Wolf, Principles of Optics, 4th ed., Pergamon Press, New York (1970).

13. Y. Z. Umul, "Scattering of waves by a half-screen with different face impedances: closed for series solution," Int. J. Electron. 100, 928-941 (2013).

14. S. Martin et al., "Starshade optical edge modeling, requirements, and laboratory tests," Proc. SPIE 8864, 88641A (2013).

15. S. Divitt, "Jreftran-a layered thin film transmission and reflection coefficient calculator," MATLAB Central File Exchange, 2020, https://www.mathworks.com/matlabcentral/ fileexchange/50923-jreftran-a-layered-thin-film-transmission-and-reflection-coefficientcalculator (accessed 27 April 2020).

Dylan McKeithen is an optical engineer at the Jet Propulsion Laboratory, where he is a member of the High Contrast Imaging Group in the Optics Section. He received his BS degree in physics from the University of Southern California in 2019.

Stuart Shaklan is the supervisor of the High Contrast Imaging Group in the Optics Section of the Jet Propulsion Laboratory. He received his PhD in optics at the University of Arizona in 1989 and has been with JPL since 1991.

David Sheikh is a chemical engineer and the founder of ZeCoat Corporation. He received his BS degree in chemical engineering at Washington University in 1987 and his MA degree in finance from Webster University in 1991.

Kunjithapatham Balasubramanian is a senior optical engineer in the High Contrast Imaging Group in the Optics Section of the Jet Propulsion Laboratory. He received his PhD in optics at the University of Arizona in 1988 and has been with JPL since 2004 after serving in other engineering leadership positions in private companies.

Eric Lowe is an undergraduate physics and mathematics major in the Morrissey College of Arts and Sciences at Boston College and was a summer intern in the Optics Section at the Jet Propulsion Laboratory in 2020. 